\title{
Construction of an Algorithm Based on Oncosis- Related LncRNAs Comprising the Molecular Subtypes and a Risk Assessment Model in Lung Adenocarcinoma
}

Guodong Xu ( $\sim$ xuguodong@nbu.edu.cn )

the Affiliated Lihuili Hospital, Ningbo University

Hang Chen

the Affiliated Lihuili Hospital, Ningbo University

Chongchang Zhou

the Affiliated Lihuili Hospital, Ningbo University

Zeyang Hu

the Affiliated Lihuili Hospital, Ningbo University

Menglu Sang

the Affiliated Lihuili Hospital, Ningbo University

Saiqi Ni

Ningbo First Hospital

Jiacheng Wu

the Affiliated Lihuili Hospital, Ningbo University

Qiaoling Pan

the Affiliated Lihuili Hospital, Ningbo University

Kaitai Liu

the Affiliated Lihuili Hospital, Ningbo University

Ni Li

the Affiliated Lihuili Hospital, Ningbo University

Linwen Zhu

the Affiliated Lihuili Hospital, Ningbo University

\section{Research Article}

Keywords: lung adenocarcinoma, oncosis-related IncRNAs, tumor immunity, prognostic signature, cluster analysis

Posted Date: February 24th, 2022 
DOI: https://doi.org/10.21203/rs.3.rs-1354579/v1

License: (c) (1) This work is licensed under a Creative Commons Attribution 4.0 International License. Read Full License 


\section{Abstract}

As an important non-apoptotic cell death method, oncosis has been reported to be closely associated with tumors in recent years. However, few researches have reported the relationship between oncosis and lung cancer. In this study, we established a oncosis-based algorithm comprised of the cluster grouping and a risk assessment model to predict the survival outcomes and related tumor immunity of patients with LUAD. We selected 11 oncosis-related IncRNAs associated with the prognosis to establish the oncosis-based algorithm and divided the LUAD patients into different clusters and different risk groups. Compared with patients in clsuter1, patients in cluster2 had a survival advantage and had a relatively more active tumor immunity. Subsequently, we constructed a risk assessment model to distinguish between patients into different risk groups, in which low-risk patients tend to have a better prognosis. GO enrichment analysis revealed that the risk assessment model was closely related to immune activities. In addition, low-risk patients tended to have higher content of immune cells and stromal cells in tumor microenvironment, higher expression of PD-1, CTLA-4, HAVCR2, and were more sensitive to ICls, including PD-1/CTLA-4 inhibitors. The risk score had a significantly positive correlation with TMB. The survival curve of the novel oncosis-based algorithm suggested that low-risk patients in cluster2 have the most obvious survival advantage. The novel oncosis-based algorithm based on the 11 oncosis-related IncRNAs was established to investigate the prognosis and the related tumor immunity of patients with LUAD, which could provide theoretical support for customized individual treatment for LUAD patients.

\section{Introduction}

As the highest incidence rate among all malignant tumors, lung cancer is considered as the primary reason of cancer-related deaths worldwide ${ }^{1}$. According to the official forecast for 2021 , lung cancer is the malignant tumor with the highest fatality rate among men and women in the United States, and its incidence rate ranks second ${ }^{2}$. According to the official forecast of China in 2015, lung cancer is the malignant tumor with the highest incidence and mortality in both men and women ${ }^{3}$. More than half of non-small cell lung cancers (NSCLC) are lung adenocarcinomas (LUAD), accounting for more than $85 \%$ of lung cancers ${ }^{4}$. Although recent breakthroughs in immunotherapy and targeted therapy have benefited a large number of advanced NSCLC patients, the 5-year survival rate for advanced NSCLC patients with distant metastasis is merely $7 \%^{5}$. Thus, identifying effective and reliable biomarkers and targets may laid a solid foundation for the individualized treatment of advanced NSCLC patients.

Manifested as DNA damage and mitochondrial swelling, oncosis is a type of programmed cell death different from apoptosis in both morphological changes and internal pathways ${ }^{6}$. The swelling of oncosis cells is mainly due to the failure of the ion pump on the membrane, which leads to water and sodium retention in the cells ${ }^{7}$. Related literature reported that oncosis might be involved in the process of tumor progression, including cancer metabolism, immunosuppression, and cancer stem cells ${ }^{8}$. In addition, studies have proved that the oncosis of cancer cells mediated by aquaporin 1 (AQP1) may be a feature of malignant tumor metastasis ${ }^{9}$. Huang et al. demonstrated that enhanced oncosis could effectively reduce 
the proliferation of bladder cancer cell lines ${ }^{10}$. Zhao et al. reported that in human esophageal squamous cell carcinoma tissues, the enhanced oncosis was caused by an insufficient blood supply, and the intensity of oncosis is closely related to apoptosis and microvessel density ${ }^{11}$. Some scholars proposed that oncosis may be the key to overcoming the main obstacle in cancer treatment-drug resistance ${ }^{12}$. In summary, oncosis has been implicated in the occurrence and development of malignant tumors.

Recent studies have shown that oncosis may be involved in immune activities. Sarode et al. suggested that cell death, including oncosis, played an important role in immune defense against infectious diseases ${ }^{13}$. Huang et al. suggested that the oncosis of $\mathrm{T}$ lymphocytes may be an effective target for the treatment of asthma ${ }^{14}$. Furthermore, related researches have demonstrated that oncosis has participated in mediating the immunogenic death of tumor cells ${ }^{15}$. Thus, we speculated that the oncosis of cancer cells was closely related to tumor immunity. In the present study, we divided the LUAD patients into different clusters and different risk groups based on the expression of the oncosis-related IncRNAs. We then analyzed the related tumor immunity between different risk groups and clusters, and explored the clinical significance of the novel oncosis-based algorithm.

\section{Materials And Methods 2.1. Data acquisition}

According to the flow chart (Fig. 1), we divided LUAD patients into different clusters and different risk groups based on the expression of orlncRNAs to predict their survival outcomes and explored the clinical significance of their prognostic signature and cluster grouping. First, we obtained LUAD patients' transcriptome data, simple nucleotide variation (SNV), and clinical data from The Cancer Genome Atlas (TCGA, https://portal.gdc.cancer.gov/) ${ }^{16}$ database. The IncRNAs and mRNAs were distinguished by gene transfer format (GTF) files. Meanwhile, a list of oncosis-related genes (orgenes) was acquired by searching for "oncosis cancer" via PubMed (https://pubmed.ncbi.nlm.nih.gov/) ${ }^{17}$. The orlncRNAs were screened by performing the Spearman correlation analysis between the orgenes and IncRNAs. The thresholds were set as $\mid$ cor $\mid>0.3$ and $p<0.001$. Furthermore, univariate Cox analysis was conducted to screen orlncRNAs closely associated with the survival of patients with LUAD $(p<0.01)$. To better illustrate the potential relationship between the orlncRNAs and orgenes, a forest map, a network diagram, and a heatmap were plotted for visualization.

\subsection{Construction of the orlncRNAs cluster}

LUAD patients were divided into different molecular subtypes to provide individualized treatment by performing consensus clustering with R-x64-4.0.3 limma, ConsensusClusterPlus packages. The heatmap clearly exhibited the expression of orlncRNAs related to prognosis and the potential relationship between clusters and clinicopathological characteristics. Then, Kaplan-Meier survival analysis was conducted to explore whether the overall survival (OS) of patients with LUAD in different clusters would be statistically different. 


\subsection{Exploration of the tumor immunity in different molecular subtypes}

The GSEA 4.0.1 (https://www.gsea-msigdb.org/gsea/index.jsp) ${ }^{18}$ was performed to find functional pathways that were probably related to the clusters. The differential expressed genes of patients in different clusters were performed on Kyoto Encyclopedia of Genes and Genomes (KEGG) ${ }^{19}$ gene sets ( $p<$ 0.05 and FDR < 0.25 ). The StromalScore, ImmuneScore, and ESTIMATEScore (StromalScore + ImmuneScore) of LUAD patients were caclulated by estimate package, and Wilcoxon rank-sum tests were conducted to explore the difference in the content of stromal cells and immune cells between different molecular subtypes. Single-sample gene-set enrichment analysis (sSGSEA) was conducted to quantify the relative abundance of various immune cells and the relative intensity of various immune activity, and multi-box plots were plotted for visualization. To investigate the expression of common ICls in different clusters, Wilcoxon rank-sum tests were performed to detect whether there were statistical differences in PD-1, PD-L1, HAVCR-2 expression between different molecular subtypes by ggpubr packages. The results above were marked as follows for visualization: $* * *<0.001, * *<0.01$, and $*<0.05$.

\subsection{Establishment of the prognostic signature}

The LUAD patients were randomly assigned into the train group and the test group evenly. Subsequently, the least absolute shrinkage and selection operator (LASSO) regression was performed to construct the optimal prognostic signature. We then exported the regression coefficient of orlncRNAs included in the modeling process and calculated every LUAD patients' risk score by the following formula:

\section{1}

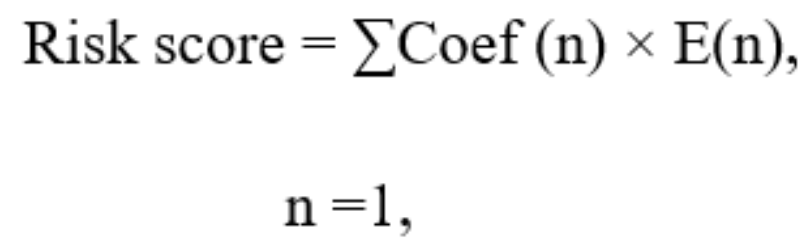

Coef $(n)$ (Table 1) and E(n) represent the regression coefficient and the expression of the orlncRNAs included by the lasso regression, respectively. The median risk score in the train group was recognized as the cut-off point of the train group and test group, thus dividing the LUAD patients into different risk groups. To clarify the correlation between molecular subtypes and risk groups, we plotted a Sankey diagram for visualization. Then, two 1-year ROC curves were plotted and the corresponding AUC values were calculated. The risk curves of the train group and test group were plotted to reveal the survival status of different risk scores. Next, Kaplan-Meier survival analyses were conducted to explore whether the OS of patients with LUAD in different risk groups was statistically significant. Two heatmaps exhibited the expression of the 11 orlncRNAs included by the lasso regression the in train group and test 
group, respectively. To investigate whether the risk assessment model was a reliable independent prognostic indicator for LUAD patients, univariate and multivariate Cox regression analyses were performed in the train group and test group, respectively, and four forest maps were generated for visualization. To better clarify the relationship between the risk groups and common clinicopathological characteristics (e.g., age, gender, Stage, T, M, N, ImmuneScore, and cluster), a clinical heatmap was plotted for visualization, which was labeled as follows: $* \star *<0.001, * *<0.01$, and $*<0.05$. To screen the enriched functional pathways, we identified the differential expressed genes with false discovery rates $($ FDR $)<0.05$ in different risk groups.

Table 1

The regression coefficients of orlnRNAs included by the lasso regression.

\begin{tabular}{|ll|}
\hline ID & Coef \\
\hline CARD8-AS1 & -0.000851001072322576 \\
\hline LINC00941 & 0.060640793514365 \\
\hline LINC01137 & 0.022200771858516 \\
\hline LINC01116 & 0.0349184545656507 \\
\hline AC010980.2 & 0.0691655030661195 \\
\hline LINC00324 & -0.158673264800938 \\
\hline AL365203.2 & 0.0358159581572812 \\
\hline AL606489.1 & 0.0412286187481897 \\
\hline AC004687.1 & -0.00643358868016261 \\
\hline HLA-DQB1-AS1 & -0.0239731776316693 \\
\hline AL590226.1 & -0.0736257017716491 \\
\hline
\end{tabular}

\subsection{Clinical application of the oncosis-based prognostic signature}

As shown through the above analyses, we demonstrated that the oncosis-based prognostic signature was indeed a reliable indicator to predict the survival outcomes of LUAD patients. We performed the GO functional enrichment analysis on the differential expressed genes $(p<0.05$ and FDR $<0.05)$ to screen the differentially expressed functional pathways between different risk groups. In the barplot, we exhibited the top ten enrichment results of biological process (BP), cellular component (CC), and molecular 
function (MF), respectively. Based on the expression of 11 orlncRNAs included by the lasso regression, we established oncosis-based molecular subtypes and a risk assessment model to evaluate the prognosis and related tumor immunity of LUAD patients. To better illustrate the potential relationship between the $T M B$, different clusters, and different risk groups, we calculated the correlation coefficient between the risk score and TMB by Spearman correlation analysis and investigated whether there were statistical differences in TMB between different risk groups, different clusters by Wilcoxon rank-sum tests. The results were exhibited by a scatter plot and two box plots. Then, we calculated the StromalScore, ImmuneScore, and ESTIMATEScore of LUAD patients and explored whether the three indicators associated with tumor microenvironment in different risk groups were statistically significant. Next, we compared the expression of common ICls (e.g., PD-1, CTLA-4, HAVCR-2) between different risk groups by Wilcoxon rank-sum tests. Immunophenoscore (IPS) was an indicator that could indirectly reflect the efficacy of PD-1 and CTLA-4 inhibitors on cancer patients. We obtained the IPS information from The Cancer Immunome Atlas (TCIA, https://tcia.at/) ${ }^{20}$ and compared the IPS between different risk groups by the Wilcoxon rank-sum tests. To prove that the combination of molecular subtypes and the risk assessment model could effectively predict the survival outcomes of LUAD patients, we divided the LUAD patients into four groups: 『cluster1 + low risk, 『cluster 1 + high risk, 『cluster 2 + low risk, 『cluster2 + high risk, and plotted a survival plot by Kaplan-Meier survival analysis.

\section{Results}

\subsection{Identification of orlncRNAs associated with OS of LUAD patients}

In the present study, all the results obtained are from samples from the TCGA database. We obtained 54 normal tissues and 497 LUAD tissues from TCGA-LUAD project. Subsequently, we collected a total of 33 orgenes by checking PubMed. Then, we identified the orlncRNAs by performing co-expression analysis between orgenes and IncRNAs. At last, 1536 orlncRNAs were screened by performing the R-x64-4.0.3 language. We screened out 41 orlncRNAs closely related to survival outcomes of LUAD patients for subsequent clustering and modeling by performing a univariate Cox analysis (Fig. 2a). A network diagram (Fig. 2b) was plotted for revealing the connection between orgenes and IncRNAs, and a heatmap (Fig. 2c) was plotted for visualizing the expression of the 41 orlncRNAs in LUAD samples and normal samples.

\subsection{Cluster grouping based on orlncRNAs in LUAD patients}

According to the expression of 41 orlncRNAs closely related to the OS of LUAD patients, we divided the LUAD patients into different clusters. In the process of generating the consensus matrix, when we set the $\mathrm{k}$ value to 2 , we got a relatively better clustering effect (Fig. 3a-c). Subsequently, we differentiated the LUAD patients into cluster1 and cluster2 based on the expression of these 41 orlncRNAs. The heatmap showed the expression of these 41 orlncRNAs, and revealed that the clusters grouping was closely 
associated with the $\mathrm{N}$ stage (Fig. 3d). Kaplan-Meier survival analysis revealed that LUAD patients in cluster2 had a greater survival advantage (Fig. 4a).

\subsection{Cluster grouping was a robust indicator of tumor immunity in LUAD patients}

GSEA analysis revealed that several basic cell activities (e.g., adherens junction, cell cycle, DNA replication, focal adhesion, proteasome, regulation of actin cytoskeleton) and several signaling pathways (e.g., p53 signaling pathway, pentose phosphate pathway, and small cell lung cancer) were enriched in cluster1 (Fig. 4b). Patients in cluster1 had a higher StromalScore (Fig. 4c), ImmuneScore (Fig. 4d), and ESTIMATEScore (Fig. 4e) than cluster2 patients, indicating that cluster1 patients had a higher content of immune cells and stromal cells in tumor microenvironment. As shown in Fig. 4f-g, common immune cells, including CD8 + T cell, dendritic cells (DCs), immature dendritic cells (iDCs), macrophages, neutrophils, nature killer (NK) cells, plasmacytoid dendritic cells (pDCs), T helper cells, follicular helper T cells (Tfh), Type 1 T helper (Th1) cells, Type 1 T helper (Th2) cells, tumor-infiltrating lymphocytes (TIL), and regulatory $T$ cells (Tregs) were more abundant in cluster1 patients, and common immune pathways, including antigen-presenting cell (APC) co-inhibition, APC co-stimulation, clinical complete response (CCR), check-point, cytolytic activity, inflammation-promoting activity, major histocompatibility complex (MHC) class I, parainflammation, T cell co-inhibition, T cell co-stimulation, and type I interferons (type I IFN) response, which were more active in cluster1 patients. Expression analyses suggested that the expression of common ICls, including PD-1 (Fig. 5a), PD-L1 (Fig. 5b), HAVCR-2 (Fig. 5c) in cluster1 patients, was significantly higher than that of cluster2 patients, which indicated that LUAD patients in cluster1 were more suitable for immunotherapy targeting PD-1, PD-L1, HAVCR-2. The results above indicated that LUAD patients in cluster1 have relatively more active tumor immunity and are more suitable for immunotherapy than cluster2 patients.

\subsection{Construction of the risk prognostic signature based on orlncRNAs in LUAD patients}

We randomly divided the 468 LUAD patients into the train group and test group evenly. Employing lasso regression, we screened out 11 orlncRNAs among 41 orlncRNAs closely associated with prognosis for subsequent modeling (Fig. 5d-e). We calculated the risk score and differentiated the patients in the train group and test group into different risk groups by the median risk score in the train group. The Sankey diagram revealed the potential relationship between clusters and risk groups, indicating that the majority of high-risk patients were in cluster1, and the majority of LUAD patients in cluster2 tended to have a lower risk score (Fig. 5f). The 1-year AUC values of the train group and test group were 0.757 (Fig. $5 \mathrm{~g}$ ) and 0.728 (Fig. 5h), respectively, which validated the predictive ability of the constructed risk model. KaplanMeier log-rank tests proved the relatively better prognosis of low-risk patients both in the train group (Fig. 6a and Fig. 6c) and test group (Fig. 6b and Fig. 6d). The heatmaps show the expression of the 11 orlncRNAs included by the lasso regression in the train group (Fig. 6e) and test group (Fig. 6f). The clinical heatmap (Fig. 7a) revealed the potential relationship between risk score and clinicopathological 
characteristics (e.g., N, T, Stage, ImmuneScore, and cluster). Univariate (Fig. 7b and Fig. 7d) and multivariate (Fig. 7c and Fig. 7e) Cox regression analysis were conducted to detect the relationship between the orlncRNA signature and clinicopathological characteristics, suggesting that the risk score (train group: hazard ratio $[\mathrm{HR}]=1.692$, confidence interval $[\mathrm{Cl}]=1.473-1.943, p<0.001$, test group: $\mathrm{HR}=$ $1.447,[\mathrm{Cl}]=1.229-1.703, p<0.001$ ) and Stage (train group: $\mathrm{HR}=1.638,[\mathrm{Cl}]=1.333-2.011, p<0.001$, test group: $\mathrm{HR}=1.478,[\mathrm{Cl}]=1.195-1.830, p<0.001$ ), were closely related to the prognosis of LUAD patients. In summary, the constructed prognostic signature could be served as independent prognostic indicators for LUAD.

\subsection{Exploration of the clinical significance of the constructed risk assessment model}

The GO enrichment analysis revealed that a majority of differentially expressed genes in different risk groups were enriched in multiple immune functions, such as immunoglobulin production, humoral immune response, etc., which indicated that the constructed risk assessment model was closely associated with tumor immunity (Fig. 8a). Therefore, we conducted detailed tumor immunity analyses of LUAD patients in different risk groups. The box plots of tumor immune microenvironment showed that the StromalScore (Fig. 8b), ImmuneScore (Fig. 8c), and ESTIMATEScore (Fig. 8d) in the low-risk group were significantly higher than that of patients with high-risk scores. The box plots revealed that low-risk patients had a higher expression of PD-1 (Fig. 8e), CTLA-4 (Fig. 8f), and HAVCR-2 (Fig. 8g). The results of IPS analyses proved the above results, indicating that low-risk patients are more sensitive to common clinical immunotherapy, including PD-1 inhibitor alone (Fig. 9a), CTLA-4 inhibitor alone (Fig. 9b), and a combination of the two (Fig. 9c). Wilcoxon signed-rank tests were performed to detect any statistical differences in TMB between different clusters and different risk groups, suggesting that TMB was positively associated with its risk score and high-risk patients or that patients in cluster1 tended to have a higher TMB (Fig. 9d-f). The survival curve exhibited the survival outcomes of the LUAD patients in different risk groups and clusters, among which low-risk patients in cluster2 have the best prognosis (Fig. 9g). As shown through the above analyses, we found that clusters had a potential relationship with risk groups, and the reasonable combination of the two might provide theoretical support for individualized treatment of advanced LUAD patients.

\section{Discussion}

Oncosis, a non-ATP-dependent active cell death, is usually an adaptive response to some form of injuries such as ischemia, hypoxia, or toxic factors ${ }^{21}$. Some research suggests that oncosis involving ROS is closely associated with acute lung injury, liver failure, and stroke ${ }^{22}$. Furthermore, the interaction between malignant tumors and oncosis has been investigated comprehensively in recent years. With the development of bioinformatics, utilizing mRNAs and IncRNAs to construct prognostic signatures for predicting the OS of cancer patients has become a hot topic of research ${ }^{23}$. We can precisely predict a 5year survival rate, the efficacy of immunotherapy, and the sensitivity to chemotherapy drugs of patients with malignant tumors by detecting the expression of a few genes or IncRNA, which provides great 
convenience for individualized clinical medication. In the present study, we divided the LUAD patients into different risk groups and different molecular subtypes based on the expression of orlncRNAs, which is a more accurate prediction than the traditional risk assessment model and is more in line with the philosophy of precision medicine.

In the present study, we collected all orgenes by searching relevant literature, obtained the orlncRNAs by conducting correlation analysis, and screened the orlncRNAs closely related to prognosis by performing univariate Cox analysis for subsequent modeling. Next, we conducted the cluster analysis to differentiate the LUAD into different clusters according to the expression of the orlncRNAs related to prognosis and found that patients in cluster2 usually had a survival advantage, while patients in cluster1 had more abundant stromal cells and immune cells, more active immune pathways, higher expression of PD-1, PDL1, HAVCR-2, and higher TMB. After randomly assigning LUAD patients into the train group and the test group, we obtained an optimal model by performing the lasso regression, calculated the risk score, performed survival analysis, and assessed the predictive ability of the prognostic signature. The results above were verified by the data of the test group. According to the $\mathrm{GO}$ enrichment analysis, we speculated that the prognostic signature was closely related to tumor immunity. After further exploration of the tumor immunity of the risk assessment model, we concluded that low-risk patients had a better survival outcome, higher expression of PD-1, CTLA-4, HAVCR-2, higher abundance of stromal cells and immune cells, and better efficacy for immunotherapy targeting PD-1, CTLA-4, while patients in the high-risk group tended to have a higher TMB. Finally, we performed survival analysis on the algorithm, which indicated that low-risk patients in cluster2 usually had the best prognosis.

In the 11 orlncRNAs included by the lasso regression, several orlncRNAs have been demonstrated to be closely associated with the progression of multiple malignant tumors. CARD8-AS1, a risk IncRNA of glioma, could regulate the metastasis of glioma cell lines ${ }^{24}$. Ren et al. demonstrated that LINC00941 acted as a sponge to interact with miR-877-3p, suppressed its expression, and promoted angiogenesis and progression of NSCLC in vitro ${ }^{25}$. In addition, LINC00941 was shown to be involved in the lung metastasis process of colorectal cancer ${ }^{26}$. Up-regulated LINC01137 enhanced the malignant tendency of oral squamous cell carcinoma cells and promoted the malignant transformation of oral cells ${ }^{27}$. LINC01116 functioned as a competing endogenous RNA (ceRNA) to bind with miR-744-5p, regulate the downstream CDCA4 expression, and accelerate proliferation, migration, invasion, and cisplatin resistance of LUAD cell lines ${ }^{28-29}$, which could be blocked by the AKT signaling pathway ${ }^{30}$. LINC00324 acted as a ceRNA to interact with miR-139-5p and down-regulated its expression, which accelerated the cell proliferation and invasion of NSCLC cell lines ${ }^{31}$. Pan et al. have also proved that LINC00324 promoted the proliferation and metastasis of LUAD tissues and cells by the miR-615-5p/AKT1 pathway ${ }^{32}$. However, in the remaining orlncRNAs of 11 orlncRNAs (e.g., AC010980.2 $2^{33-36}$, AL365203.2 ${ }^{37-39}$, AL606489.1 40,34, AC004687. $1^{41-43}$, HLA-DQB1-AS1 ${ }^{40,47}$, AL590226. ${ }^{48}$ ), although there are few experiments to explore their relationship with the occurrence and development of malignant tumors, plenty of prognostic signatures have included them to predict the OS of patients with various malignant tumors. 
We are the first to establish a novel oncosis-based algorithm and explore the clinical significance of the risk assessment model and molecular subtypes. Based on the expression of orlncRNAs, we classified the LUAD patients twice, namely cluster analysis combined with multivariate Cox regression analysis, which is relatively better than traditional modeling procedures for individualized management on LUAD patients. In addition, we analyzed the survival outcome and tumor immunity of LUAD patients in different risk groups and different clusters, respectively, which could provide solid theoretical support for precision medicine.

However, there are several limitations in our study. Firstly, given that the data was obtained from the open public database, the bias of the profile analyzed should not be neglected. Secondly, we utilized the data from TCGA database to perform internal verification for the constructed risk assessment model, rather than conducting external verification by using data sets other than TCGA database. Thirdly, the novel algorithm ultimately served for clinical treatment. Whether the predictive ability of the algorithm in the clinical treatment of LUAD patients is as accurate as analyzed, this needs be further verified by qRT-PCR.

In conclusion, we established a novel algorithm that combined cluster analysis with multivariate Cox regression analysis. We only needed to detect the 11 IncRNAs expression to distinguish LUAD patients into different molecular subtypes and risk groups, to predict the survival outcomes and related tumor immunity, which could provide individualized management and treatment for LUAD patients.

\section{Declarations}

\section{Ethics approval and consent to participants}

Not applicable

\section{Consent for publication}

Written informed consent for publication was obtained from all participants.

\section{Data availability}

All data analysed during the current study are accessible from the TCGA database(https://portal.gdc.cancer.gov/).

\section{Funding}

The study was funded by Basic public welfare project of Ningbo (Grant No. 2019C50041), The Natural Science Foundation of Zhejiang $₫$ Grant No. LY22H160004】 


\section{Author contribution}

Hang Chen and Guodong Xu contributed to the conception of the study;

Chongchang Zhou, Zeyang Hu, Menglu Sang and Saiqi Ni performed the R language;

Jiacheng Wu and Qiaoling Pan contributed significantly to analysis and manuscript preparation; Hang Chen performed the data analyses and wrote the manuscript;

Kaitai Liu, Ni Li, and Linwen Zhu helped perform the analysis with constructive discussions.

\section{Conflict of interest}

The authors declare that they have no conflicts of interest.

\section{Acknowledgement}

We thank the TCGA database for generously sharing a large amount of data.

\section{References}

1. Nasim, F., Sabath, B., Eapen, G. A. (2019). Lung cancer. The Medical Clinics of North America, 103(3), 463-473.

2. Siegel, R., Miller, K., Fuchs, H., Jemal, A. (2021). Cancer statistics, 2021. CA: A Cancer Journal for Clinicians, 71(1), 7-33.

3. Chen, W., Zheng, R., Baade, P., Zhang, S., Zeng, H., Bray, F., Jemal, A., Yu, X., He, J. (2016). Cancer statistics in China, 2015. CA: A Cancer Journal for Clinicians, 66(2), 115-32.

4. Shi, J., Wang, L., Wu, N., Li, J., Hui, Z., Liu, S., Yang, B., Gao, S., Ren, J., Huang, H., Zhu, J., Liu, C., Fan, J., Zhao, S., Xing, P., Zhang, Y., Li, N., Lei, W., Wang, D., Huang, Y., Liao, X., Xing, X., Du, L., Yang, L., Liu, Y., Zhang, Y., Zhang, K., Qiao, Y., He, J., Dai, M. (2019). Clinical characteristics and medical service utilization of lung cancer in China, 2005-2014: Overall design and results from a multicenter retrospective epidemiologic survey. Lung Cancer, 128: 91-100.

5. American Cancer Society. Non-small cell lung cancer survival rates by stage[OL]. .Date last updated: 04/20/21.

6. Wang, K., Shao, X., Tian, Z., Liu, L., Zhang, C., Tan, C., Zhang, J., Ling, P., Liu, F., Chen, Q., Diao, J., Mao, Z. (2021). A Continuous Add-On Probe Reveals the Nonlinear Enlargement of Mitochondria in Light- 
Activated Oncosis. Advancement of Science, e2004566.

7. Majno, G., Joris, I. (1995). Apoptosis, oncosis, and necrosis. An overview of cell death. American Journaol of Pathology. 146(1): 3-15.

8. Xu, C., Zhang, H., Mu, L., Yang, X. (2020). Artemisinins as Anticancer Drugs: Novel Therapeutic Approaches, Molecular Mechanisms, and Clinical Trials. Frontiers in Pharmacology, 11: 529881.

9. Tsai, C. (2020). AqF026 may act as a cancer therapeutic agent via inducing cancer cell oncosis. Medical Hypotheses, 140: 109685.

10. Huang, J., Wu, Z., Wang, G., Cai, Y., Cai, M., Li, Y. (2015). N-Myc downstream-regulated gene 2 suppresses the proliferation of T24 human bladder cancer cells via induction of oncosis. Molecular Medicine Reports, 12(4): 5730-6.

11. Zhao, G., Seng, J., Zhao, S., Hu, W., Li, A., Li, X., Qi, Y. (2007). Oncosis in human esophageal squamous cell carcinoma and its relationship with apoptosis and microvessel density. Chinese Medical Journal, 120(22): 1999-2001.

12. Guan, R., Chen, Y., Zeng, L. (2018). Oncosis-inducing cyclometalated iridium(iii) complexes. Chemical Science, 9(23): 5183-90.

13. Sarode, G., Jain, P., Sengupta, N., Sarode, S., Patil, S. (2019). Pyroptosis and Oral Cancer. The Journal of Contemporary Dental Practice, 20(9): 1001-2.

14. Huang, X., He, J., Liu, M., Zhou, C. (2014). The influence of aminophylline on the nanostructure and nanomechanics of T lymphocytes: an AFM study. Nanoscale Research Letters, 9(1): 518.

15. O'Cathail, S., Pokrovska, T., Maughan, T., Fisher, K., Seymour, L., Hawkins M. (2017). Combining Oncolytic Adenovirus with Radiation-A Paradigm for the Future of Radiosensitization. Frontiers in Oncology, 7: 153.

16. Tomczak, K., Czerwińska, P., Wiznerowicz, M. (2015). The Cancer Genome Atlas (TCGA): an immeasurable source of knowledge. Contemporary Oncology, 19: A68-77.

17. Fiorini, N., Lipman, D., Lu, Z. (2017). Towards PubMed 2.0. Elife, 6.

18. Subramanian, A., Tamayo, P., Mootha, V., Mukherjee, S., Ebert, B., Gillette, M., Paulovich, A., Pomeroy, S., Golub, T., Lander, E., Mesirov, J. (2005). Gene set enrichment analysis: a knowledge-based approach for interpreting genome-wide expression profiles. Proceedings of the National Academy of Sciences of the United States of America, 102(43): 15545-50.

19. Kanehisa, M., Goto, S. (2000). KEGG: kyoto encyclopedia of genes and genomes. Nucleic Acids Research, 28(1): 27-30.

20. Prior, F., Clark, K., Commean, P., Freymann, J., Jaffe, C., Kirby, J., Moore, S., Smith, K., Tarbox, L., Vendt, B., Marquez, G. (2013). TCIA: An information resource to enable open science. Annual International Conference of the IEEE Engineering Medicine and Biology Society, 2013: 1282-5.

21. Moinfar, F., Mannion, C., Man, Y., Tavassoli, F. (2000). Mammary "comedo"-DCIS: apoptosis, oncosis, and necrosis: an electron microscopic examination of 8 cases. Ultrastructural Pathology, 24(3): 13544. 
22. Yu, J., Zhong, B., Xiao, Q., Du, L., Hou, Y., Sun, H., Lu, J., Chen, X. (2020). Induction of programmed necrosis: A novel anti-cancer strategy for natural compounds. Clinical Pharmacology \& Therapeutics, 214: 107593.

23. Chen, H., Shen, W., Ni, S., Sang, M., Wu, S., Mu, Y., Liu, K., Li, N., Zhu, L., Xu, G. (2021). Construction of an immune-related IncRNA signature as a novel prognosis biomarker for LUAD. Aging, 13.

24. Lin, X., Jiang, T., Bai, J., Li, J., Wang, T., Xiao, J., Tian, Y., Jin, X., Shao, T., Xu, J., Chen, L., Wang, L., Li, Y. (2018). Characterization of Transcriptome Transition Associates Long Noncoding RNAs with Glioma Progression. Molecular Therapy-Nucleic Acids, 13: 620-32.

25. Ren, M., Chen, S., Wang, L., Rui, W., Li, P. (2021). LINC00941 Promotes Progression of Non-Small Cell Lung Cancer by Sponging miR-877-3p to Regulate VEGFA Expression. Frontiers in Oncology, 11: 650037.

26. Wu, N., Jiang, M., Liu, H., Chu, Y., Wang, D., Cao, J., Wang, Z., Xie, X., Han, Y., Xu, B. (2021). LINC00941 promotes $\mathrm{CRC}$ metastasis through preventing SMAD4 protein degradation and activating the TGF$\beta / S M A D 2 / 3$ signaling pathway. Cell Death and Differentiation, 28(1): 219-32.

27. Du, Y., Yang, H., Li, Y., Guo, W., Zhang, Y., Shen, H., Xing, L., Li, Y., Wu, W., Zhang, X. (2021). Long noncoding RNA LINC01137 contributes to oral squamous cell carcinoma development and is negatively regulated by miR-22-3p. Cellular oncology, 44(3): 595-609.

28. Ren, P., Chang, L., Hong, X., Xing, L., Zhang, H. (2021). Long non-coding RNA LINC01116 is activated by EGR1 and facilitates lung adenocarcinoma oncogenicity via targeting miR-744-5p/CDCA4 axis. Cancer Cell International, 21(1): 292.

29. Wang, J., Gao, J., Chen, Q. Zou, W., Yang, F., Wei, C., Wang, Z. (2020). LncRNA LINC01116 Contributes to Cisplatin Resistance in Lung Adenocarcinoma. OncoTargets and Therapy, 13: 9333-48.

30. Shang, B., Li, Z., Li, M., Jiang, S., Feng, Z., Cao, Z., Wang, H. (2021). Silencing LINC01116 suppresses the development of lung adenocarcinoma via the AKT signaling pathway. Thoracic Cancer, 12(14): 2093-103.

31. Zhang, M., Lin, B., Liu, Y., Huang, T., Chen, M., Lian, D., Deng, S., Zhuang, C. (2020). LINC00324 affects non-small cell lung cancer cell proliferation and invasion through regulation of the miR-1395p/IGF1R axis. Molecular and Cellular Biochemistry, 473: 193-202.

32. Pan, Z., Guo, X., Shan, J., Luo, S. (2018). LINC00324 exerts tumor-promoting functions in lung adenocarcinoma via targeting miR-615-5p/AKT1 axis. European Review for Medical and Pharmacological Sciences, 22(23): 8333-42.

33. Shao, J., Zhang, B., Kuai, L., Li, Q. (2021). Integrated analysis of hypoxia-associated IncRNA signature to predict prognosis and immune microenvironment of lung adenocarcinoma patients. Bioengineered, 12(1): 6186-200.

34. Guo, Y., Qu, Z., Li, D., Bai, F., Xing, J., Ding, Q., Zhou, J., Yao, L., Xu, Q. (2021). Identification of a prognostic ferroptosis-related IncRNA signature in the tumor microenvironment of lung adenocarcinoma. Cell Death Discovery, 7(1): 190. 
35. Wu, L., Wen, Z., Song, Y., Wang, L. (2021). A novel autophagy-related IncRNA survival model for lung adenocarcinoma. Journal of Cellular and Molecular Medicine, 25(12): 5681-90.

36. You, J., Fang, W., Zhao, Q., Chen, L., Chen, L., Chen, F. (2021). Identification of a RNA-Seq Based Prognostic Signature with Seven Immune-Related IncRNAs for Lung Adenocarcinoma. Clinical Laboratory, 67(3).

37. Xu, B., Jiang, J., Luo, T., Jiang, Z., Liu, X., Li, L. (2021). Signature of prognostic epithelialmesenchymal transition-related long noncoding RNAs (ERLs) in hepatocellular carcinoma. Medicine, 100(30): e26762.

38. Zhou, P., Lu, Y., Zhang, Y., Wang, L. (2021). Construction of an Immune-Related Six-IncRNA Signature to Predict the Outcomes, Immune Cell Infiltration, and Immunotherapy Response in Patients With Hepatocellular Carcinoma. Frontiers in Oncology, 11: 661758.

39. Jia, Y., Chen, Y., Liu, J. (2020). Prognosis-Predictive Signature and Nomogram Based on AutophagyRelated Long Non-coding RNAs for Hepatocellular Carcinoma. Frontiers in Genetics, 11: 608668.

40. Liu, B., Yang, S. (2021). A Five Autophagy-Related Long Non-Coding RNA Prognostic Model for Patients with Lung Adenocarcinoma. International Journal of General Medicine, 14: 7145-58.

41. Cai, H., Zhuang, Z., Wu, Y., Zhang, Y., Liu, X., Zhuang, J., Yang, Y., Gao, Y., Chen, B., Guan, G. (2021). Development and validation of a ferroptosis-related IncRNAs prognosis signature in colon cancer. Bosnian Journal of Basic Medical Sciences.

42. Huang, S., Li, G., Zhou, X., Chen, X., Yao, Y., Zhang, X., Liang, Y., Li, M., Chen, G., Huang, Z., Dang, Y., Li, J., Li, P., Tang, X., Rong, M. (2020). Identification of an Immune Score-Based Gene Panel with Prognostic Power for Oral Squamous Cell Carcinoma. Medical Science Monitor, 26: e922854.

43. Ye, J., Wu, S., Pan, S., Huang, J., Ge, L. (2020). Risk scoring based on expression of long non-coding RNAs can effectively predict survival in hepatocellular carcinoma patients with or without fibrosis. Oncology Reports, 43(5): 1451-66.

44. Xue, L., Wu, P., Zhao, X., Jin X, Wang, J., Shi, Y., Yang, X., She, Y., Li, Y., Li, C. (2021). Using ImmuneRelated IncRNA Signature for Prognosis and Response to Immunotherapy in Cutaneous Melanoma. International Journal of General Medicine, 14: 6463-75.

45. Ding, Y., Li, T., Li, M., Tayier, T., Zhang, M., Chen, L., Feng, S. (2021). A Novel Autophagy-Related IncRNA Gene Signature to Improve the Prognosis of Patients with Melanoma. Biomed Research International, 2021: 8848227.

46. Jin, D., Song, Y., Chen, Y., Zhang, P. (2020). Identification of a Seven-IncRNA Immune Risk Signature and Construction of a Predictive Nomogram for Lung Adenocarcinoma. Biomed Research International, 2020: 7929132.

47. Qian, Y., Mao, Z., Shi, Y., Liu, Z., Cao, Q., Zhang, Q. (2018). Comprehensive Analysis of miRNA-mRNAIncRNA Networks in Non-Smoking and Smoking Patients with Chronic Obstructive Pulmonary Disease. Cellular Physiology and Biochemistry, 50(3): 1140-53.

48. Geng, W., Lv, Z., Fan, J., Xu, J., Mao, K., Yin, Z., Qing, W., Jin, Y. (2021). Identification of the Prognostic Significance of Somatic Mutation-Derived LncRNA Signatures of Genomic Instability in Lung 


\section{Figures}

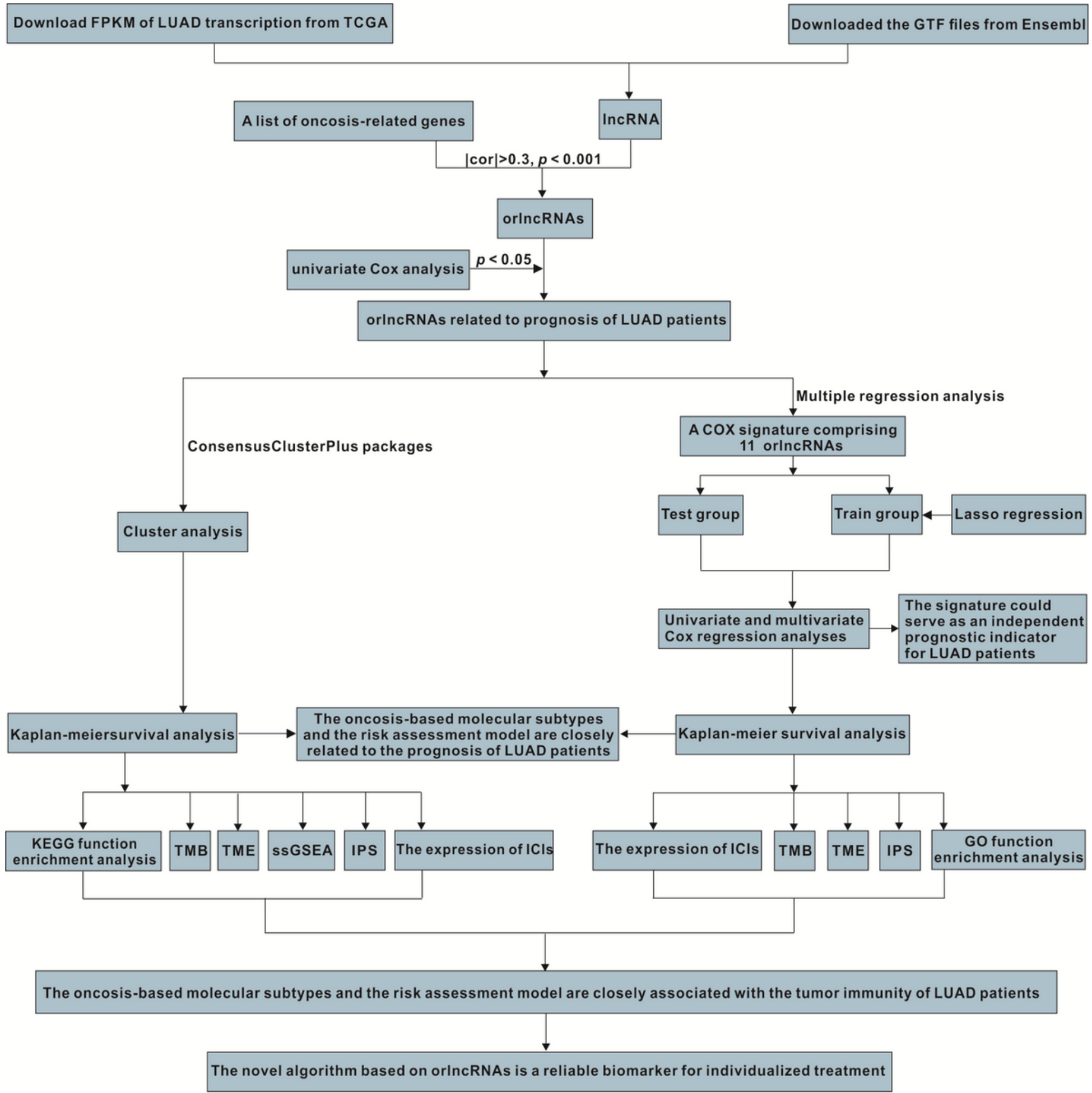

\section{Figure 1}

The flowchart for constructing a novel oncosis-based algorithm and its implication. 

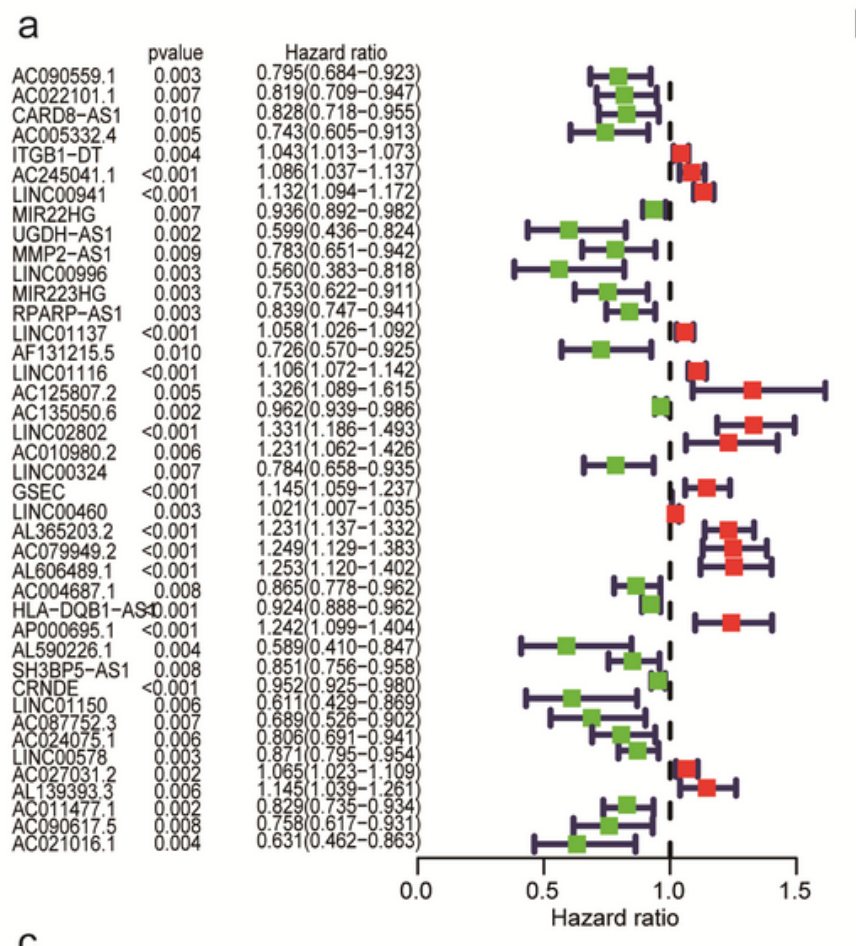

b

C

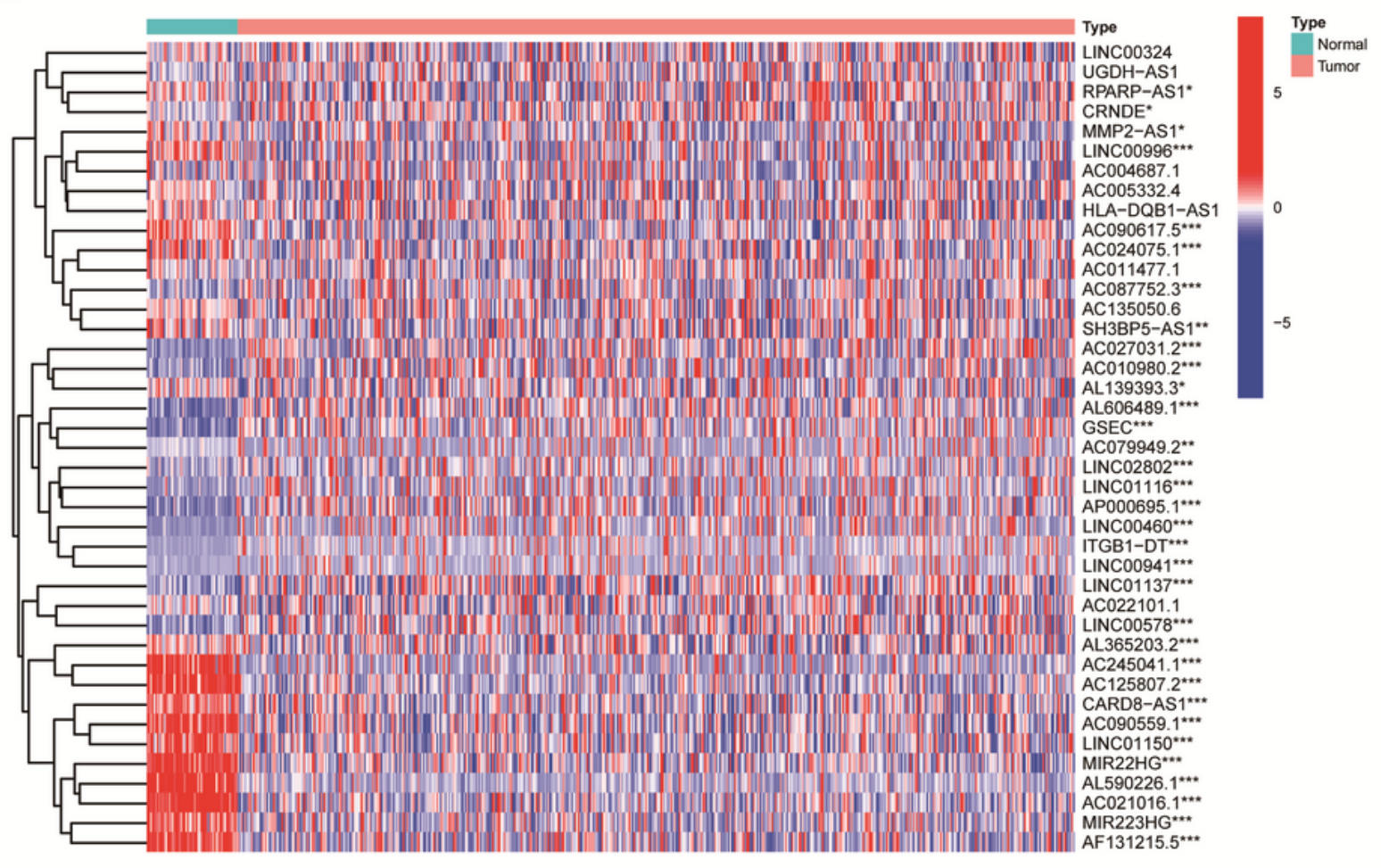

Figure 2

Identification of the orlncRNAs closely associated with the prognosis of LUAD patients. (a) The univariate Cox analysis identifies the 41 orlncRNAs closely related to the survival outcomes of LUAD patients. Red marks represent risk IncRNAs, while green represent protective IncRNAs. (b) The network diagram exhibits the connection between the orgenes and IncRNAs. The blue oval represents IncRNAs, and the pink 
rectangle represents oncosis-related mRNA. (c) The heatmap shows the expression of the 41 orlncRNAs in LUAD samples and normal samples.

a

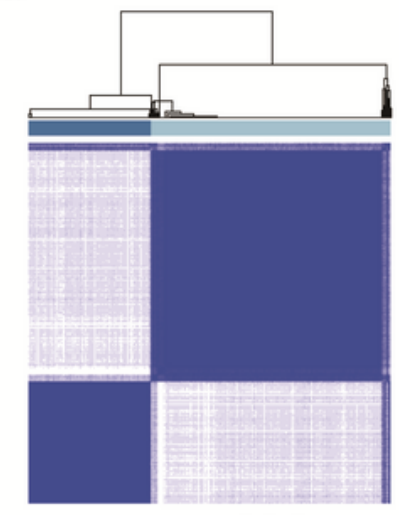

consensus matrix $\mathrm{k}=2$ b

\section{\begin{tabular}{|l|}
\hline 몸 \\
\hline
\end{tabular}}

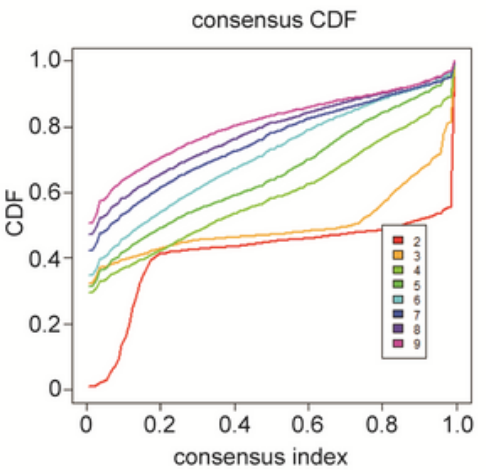

C

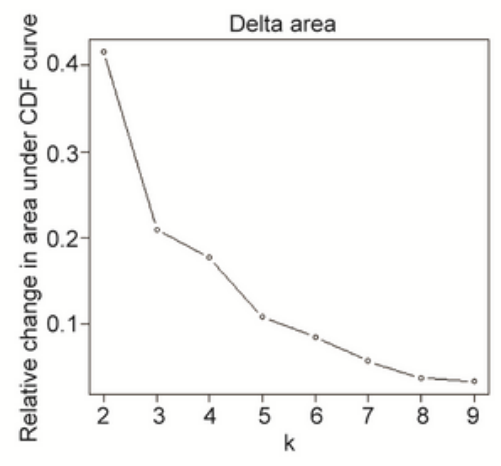

d

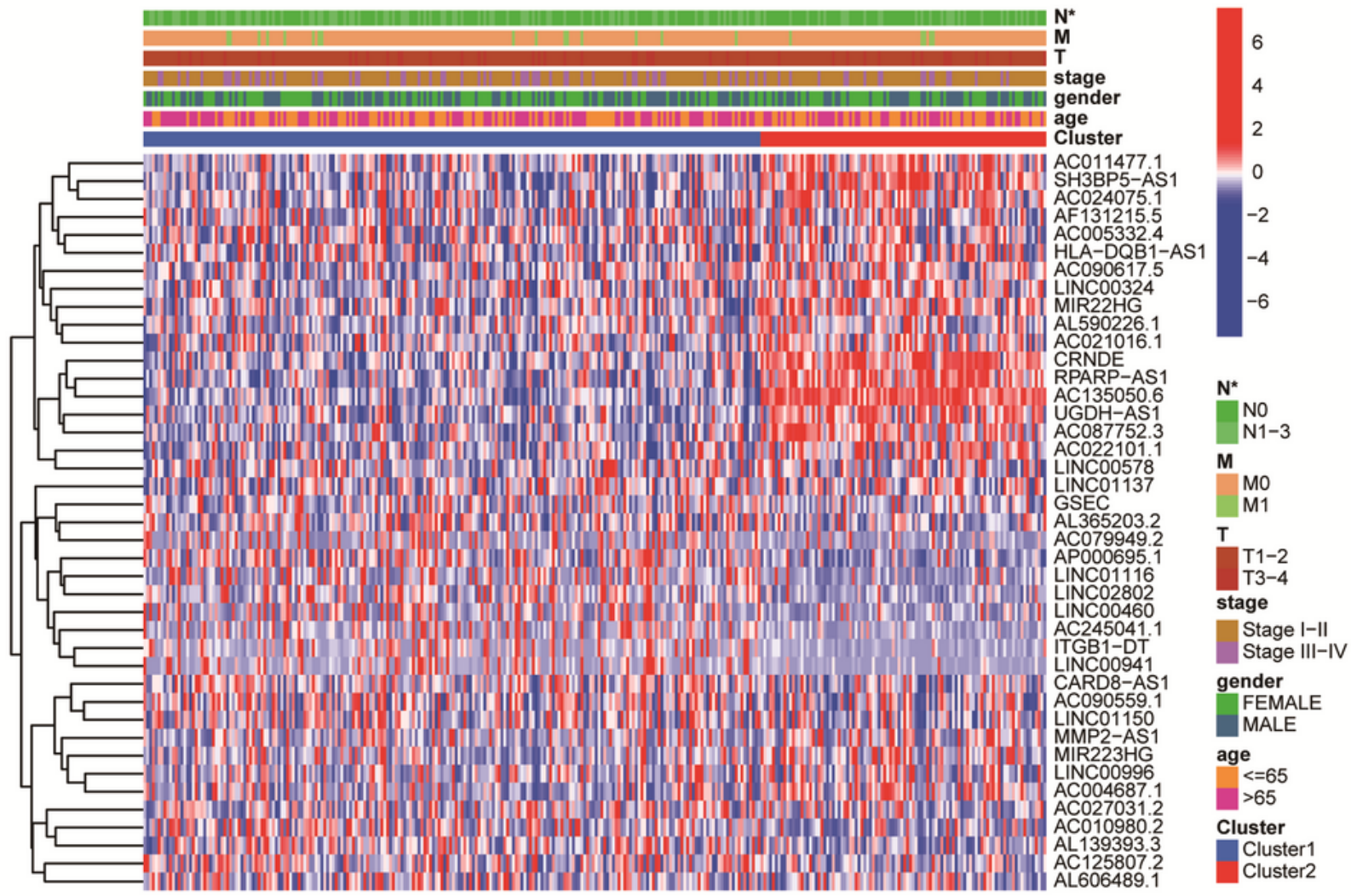

Figure 3

Cluster analysis of LUAD patients based on the expression of the 41 orlncRNAs. (a-c) In the process of generating the consensus matrix, when we set the $k$ value to 2 , the effect of large differences between 
clusters, but small differences within the cluster is the best (a), the descending slope of cation diffusion facilitator (CDF) is the smallest (b), the delta area under CDF curve is the biggest (c), which indicates that we obtained a relatively better clustering effect at this time. (d) The heatmap exhibits the expression of the 41 orlncRNAs in different clusters and reveals that the clusters grouping is closely associated with the N.

a

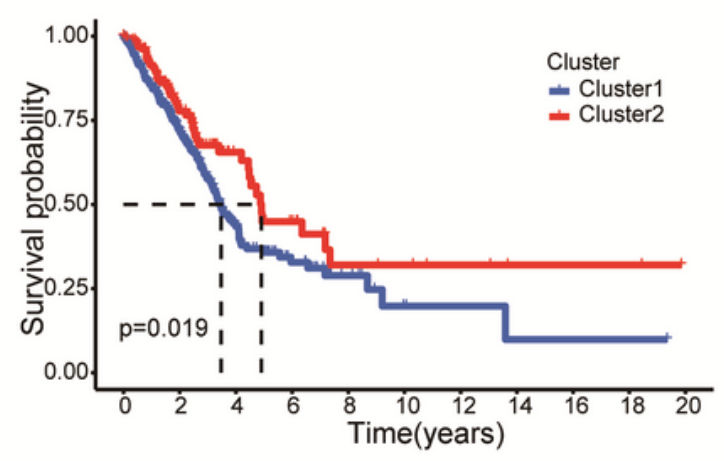

Number at risk

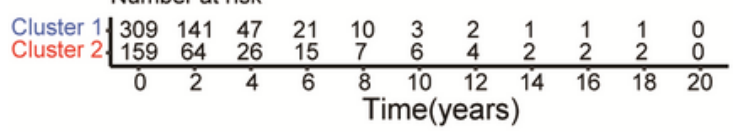

b

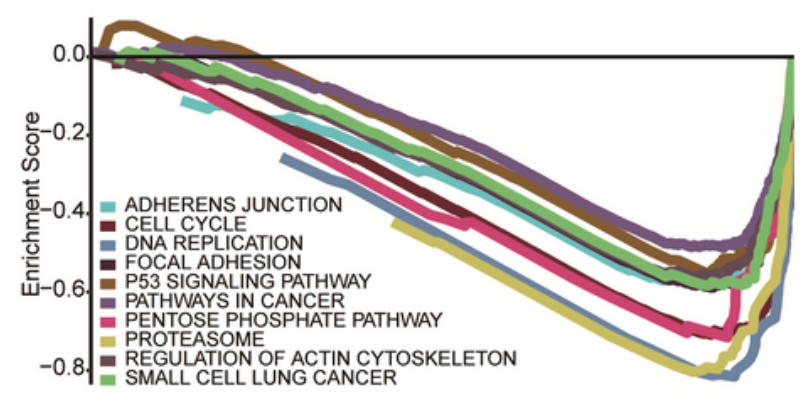

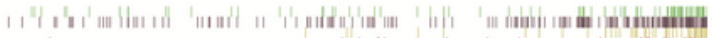

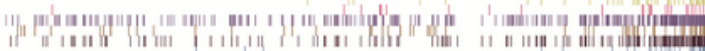

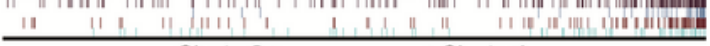
Cluster2<----------->Cluster1
C Type 申Cluster1 申 Cluster2

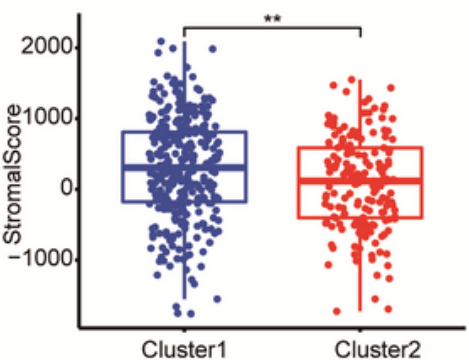

f

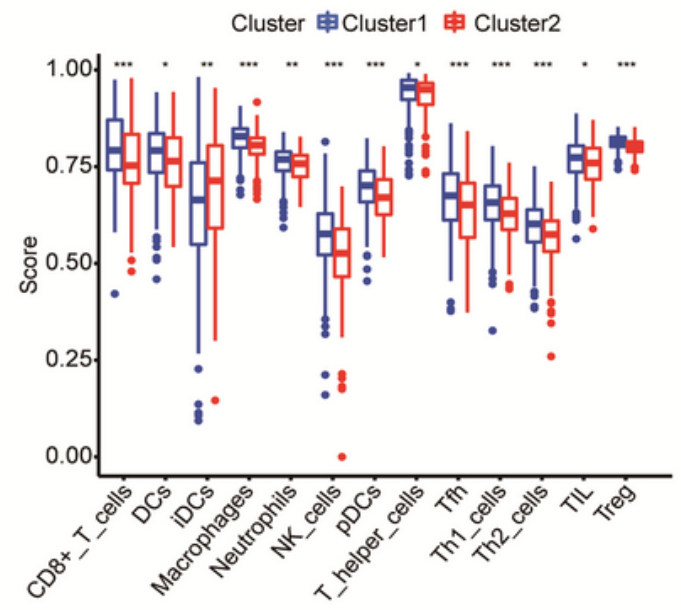

Type 申Cluster1 申 Cluster2
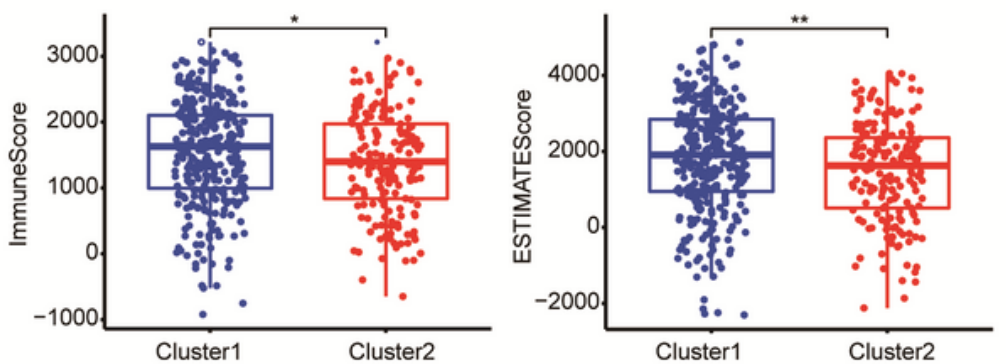

g

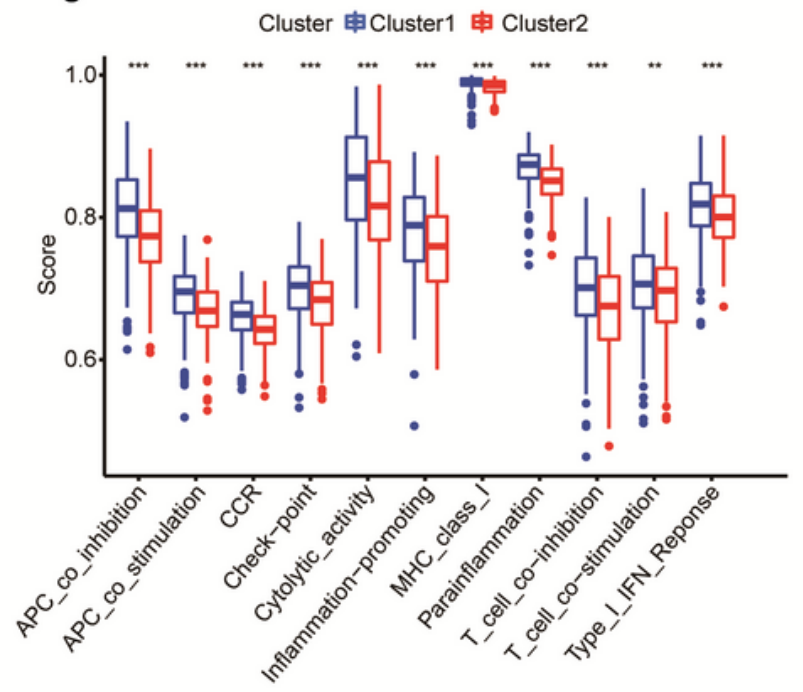

Figure 4 
The survival and related tumor immunity of LUAD patients between different clusters. (a) The KaplanMeier survival analysis indicates that patients in cluster2 have relatively better survival outcomes. (b) GSEA analysis reveals that several basic cell activities and signal pathways are enriched in cluster1. (c-e) The box plots suggested that patients in cluster1 have a relatively higher StromalScore (c), ImmuneScore (d), and ESTIMATEScore (e). ( $\mathrm{f}$ and $\mathrm{g}$ ) The multi-box plots indicate that common immune cells are more abundant in cluster1 patients (f), and common immune pathways are more active in cluster1 patients (g).

a

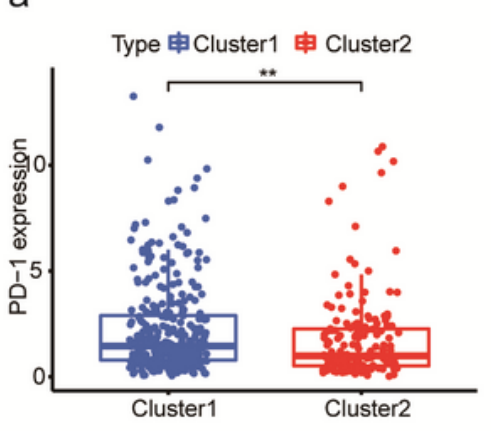

d

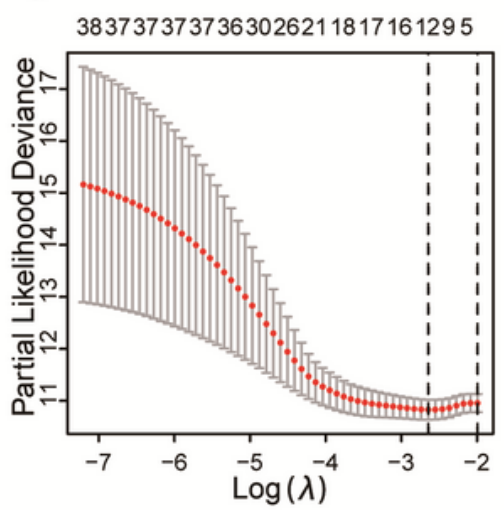

b

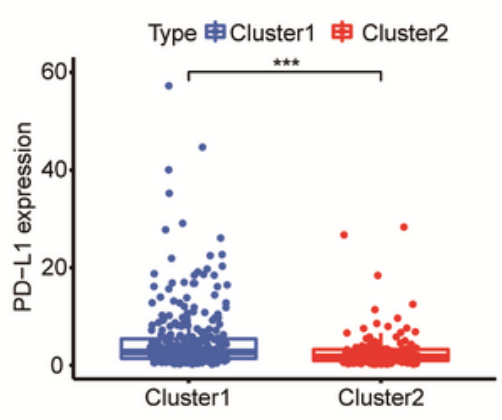

C

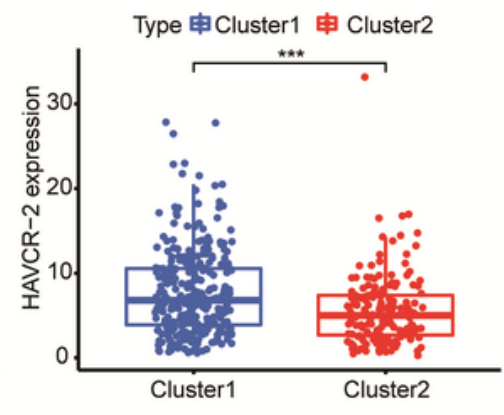

f

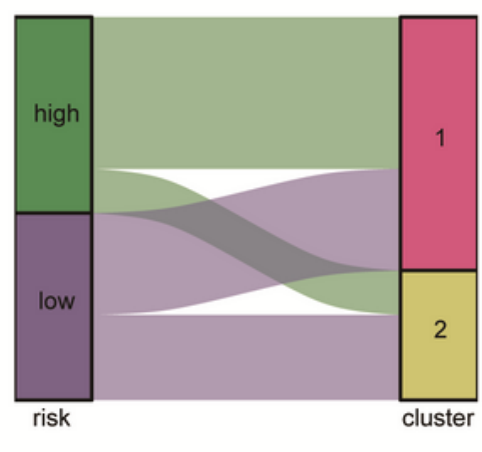

g

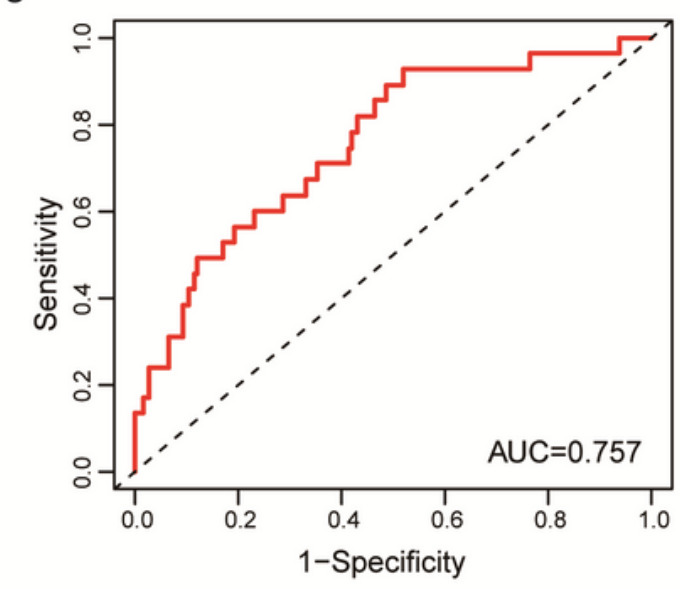

h

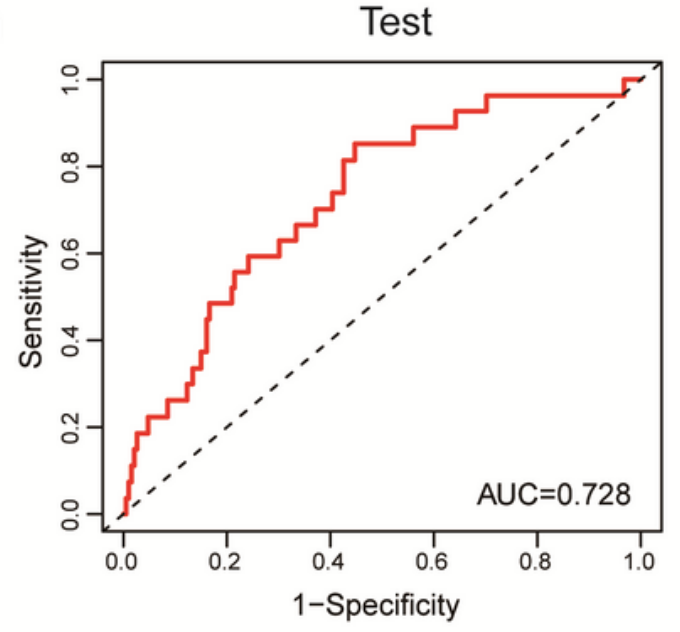




\section{Figure 5}

Construction and Validation of the 11 orlncRNAs signature. (a-c) The box plots indicated that the expression of common ICls, including PD-1 (a), PD-L1 (b), HAVCR-2 (c) in cluster1 patients, were significantly higher than those in cluster2 patients. (c) ( $d$ and e) Lasso regression was performed on patients in the train group. (f) The Sankey diagram suggests that a majority of high-risk patients were in cluster1, and the majority of LUAD patients in cluster2 tended to have a lower risk score. ( $\mathrm{g}$ and $\mathrm{h}$ ) The 1year ROC curves indicate that the predictive ability of the train group $(\mathrm{g})$ and test group $(\mathrm{h})$ are satisfactory. 


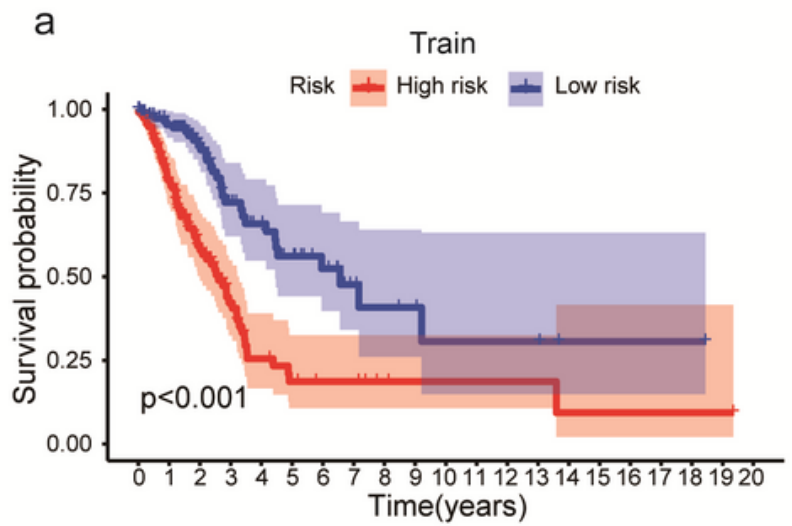

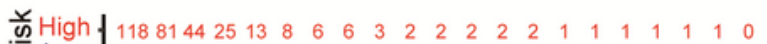

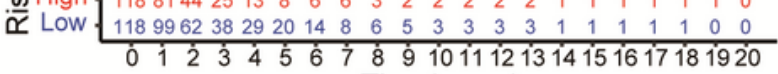
Time(years)

C
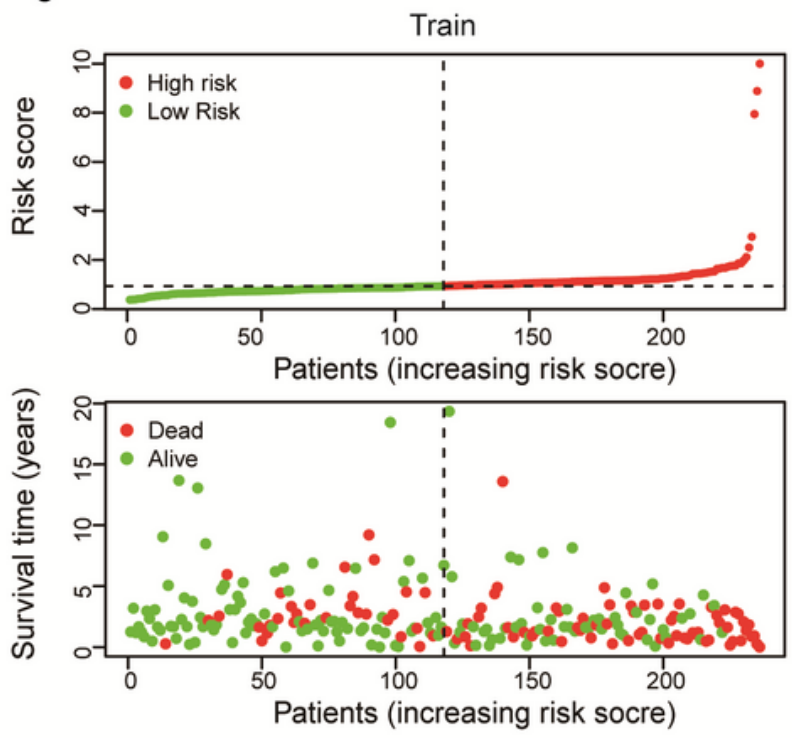

e

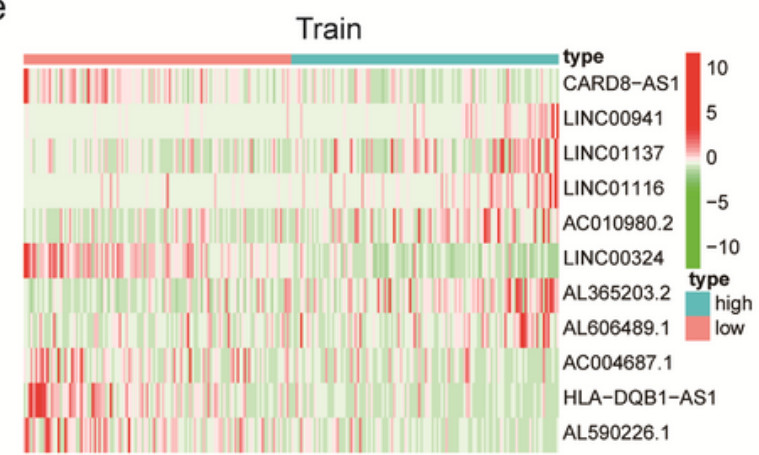

b

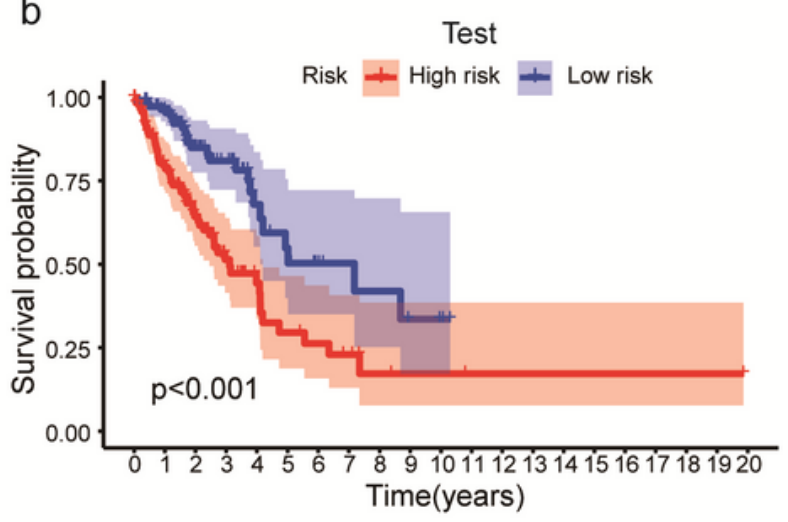

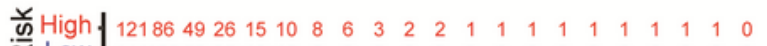

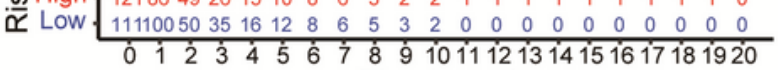
Time(years)

d
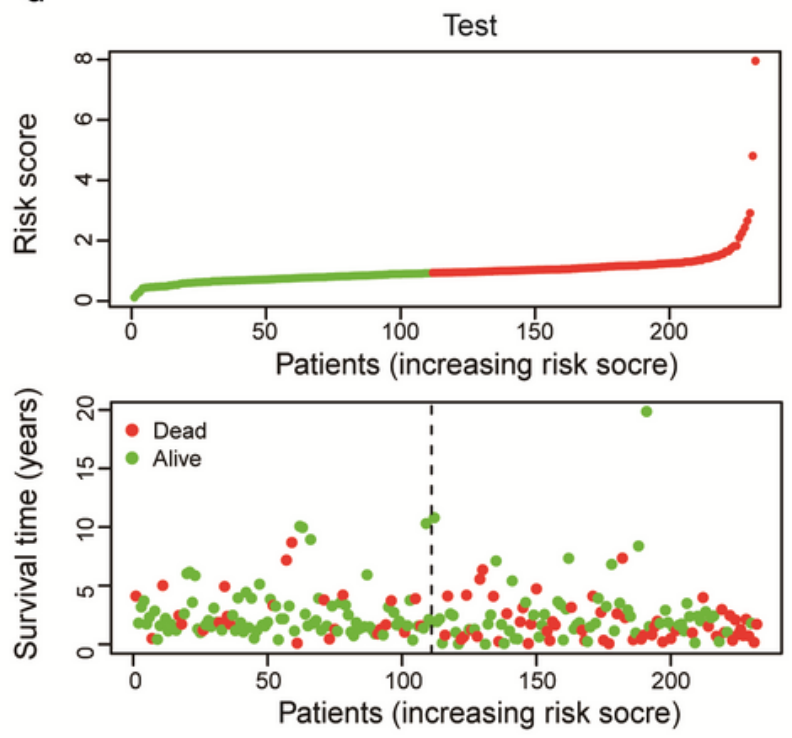

f

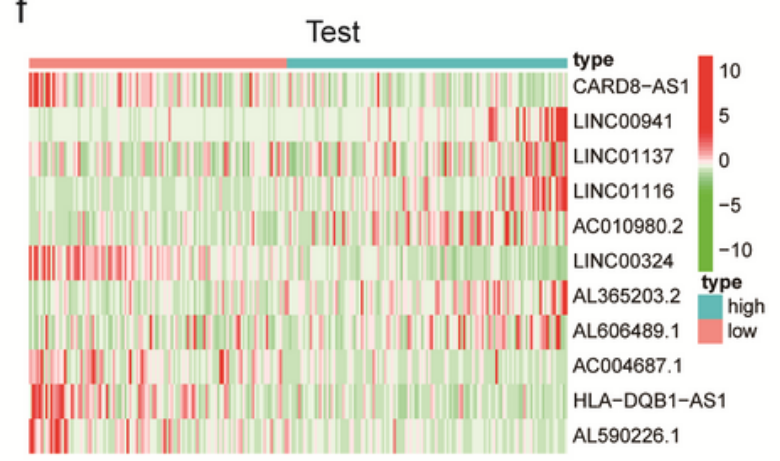

\section{Figure 6}

Validation of the 11 orlncRNAs signature. (a-d) The survival curves (a and b) and scatter plots (c and d) indicate that the low-risk patients in the train group ( $a$ and $c$ ) and test group (b and d) exhibit relatively better survival outcomes. The heatmaps show the expression of the 11 orlncRNAs included by the lasso regression in the train group (e) and test group (f). 
a

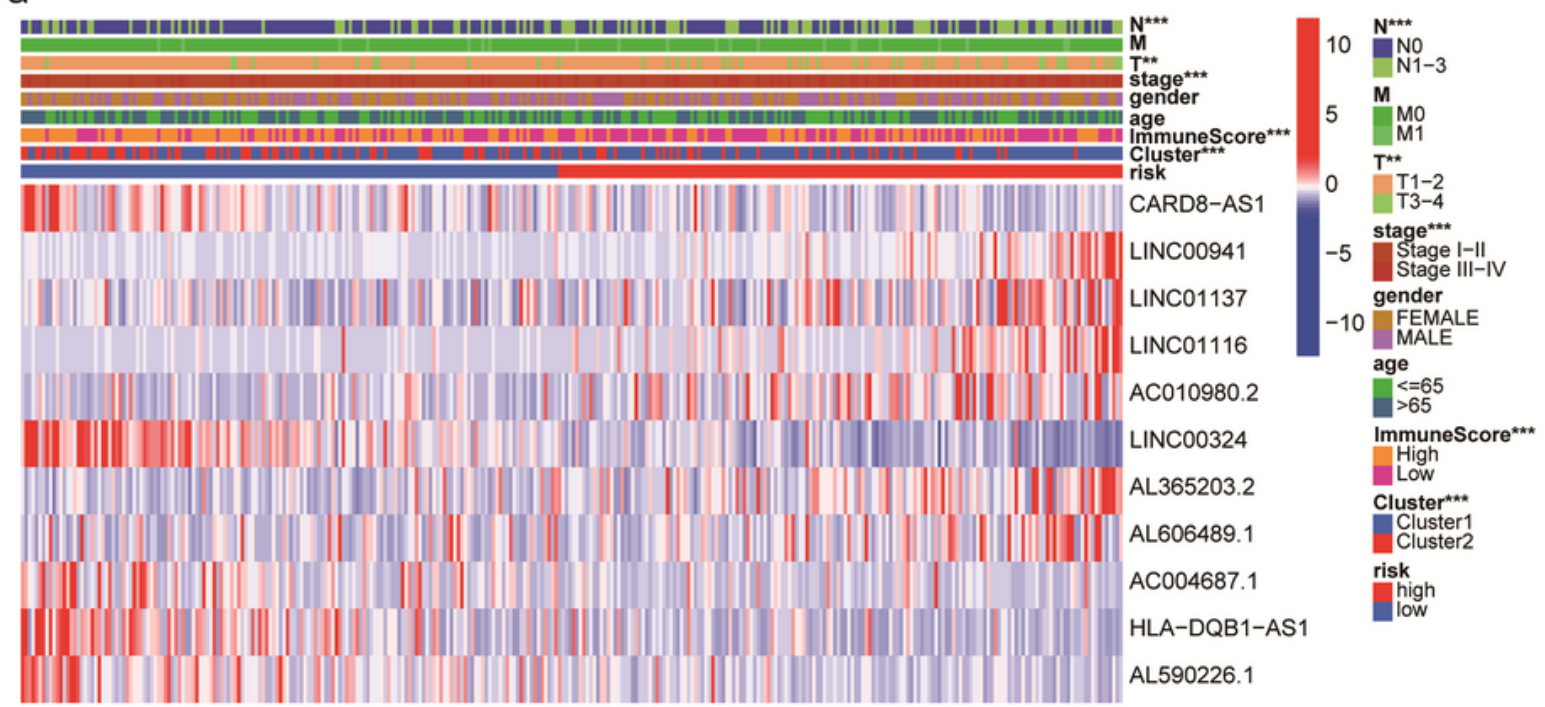

b

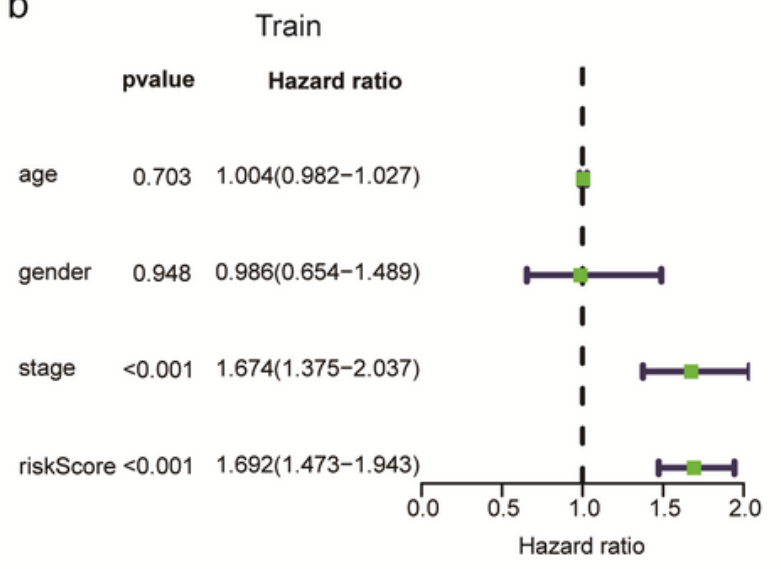

d

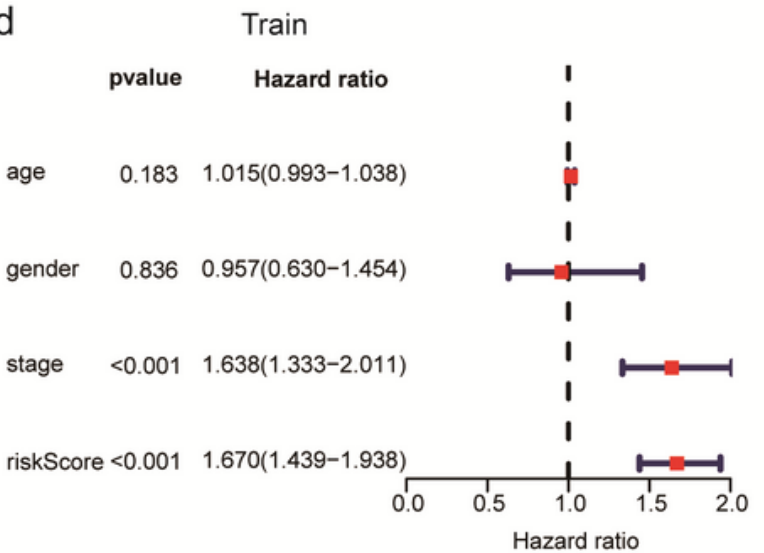

C

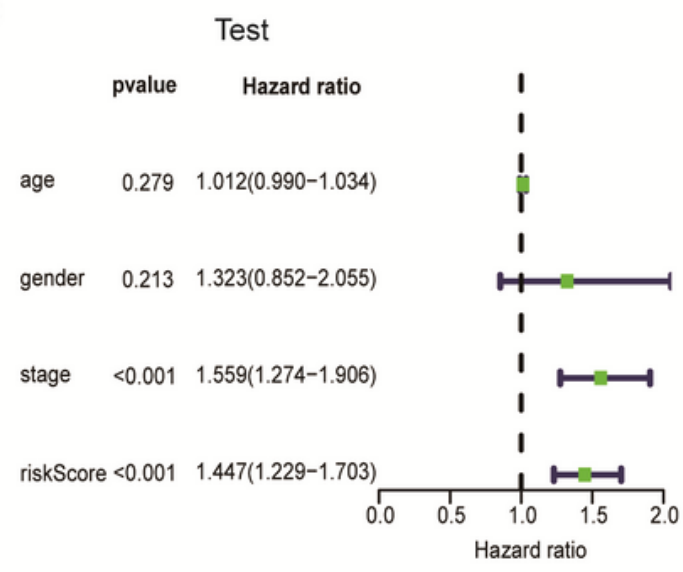

e

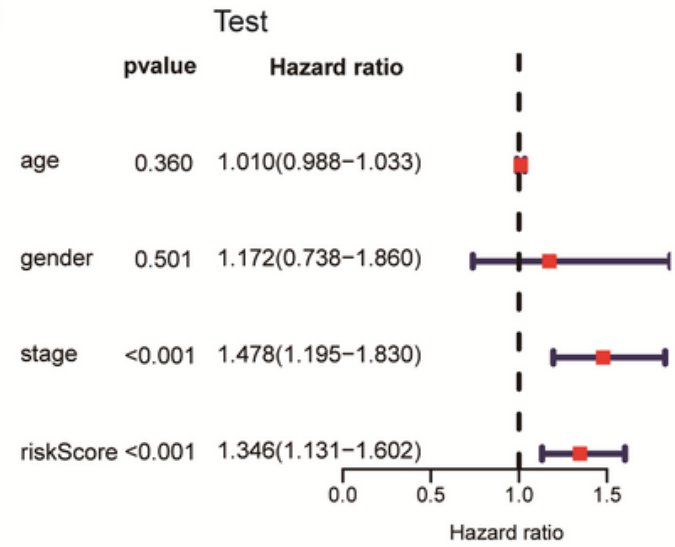

\section{Figure 7}

The oncosis-based prognostic signature is a reliable biomarker for LUAD patients. (a) The clinical heatmap indicates that the risk score is closely associated with N, T, Stage, ImmuneScore, and cluster. (be) The forest maps suggested that risk score (train group: hazard ratio $[H R]=1.692$, confidence interval $[\mathrm{Cl}]=1.473-1.943, p<0.001$, test group: $\mathrm{HR}=1.447,[\mathrm{Cl}]=1.229-1.703, p<0.001$ ) and Stage (train group: $\mathrm{HR}=1.638,[\mathrm{Cl}]=1.333-2.011, p<0.001$, test group: $\mathrm{HR}=1.478,[\mathrm{Cl}]=1.195-1.830, p<0.001)$, 
could serve as an independent prognostic indicator for LUAD patients in the train group (b and d) and test group (c and e).

a

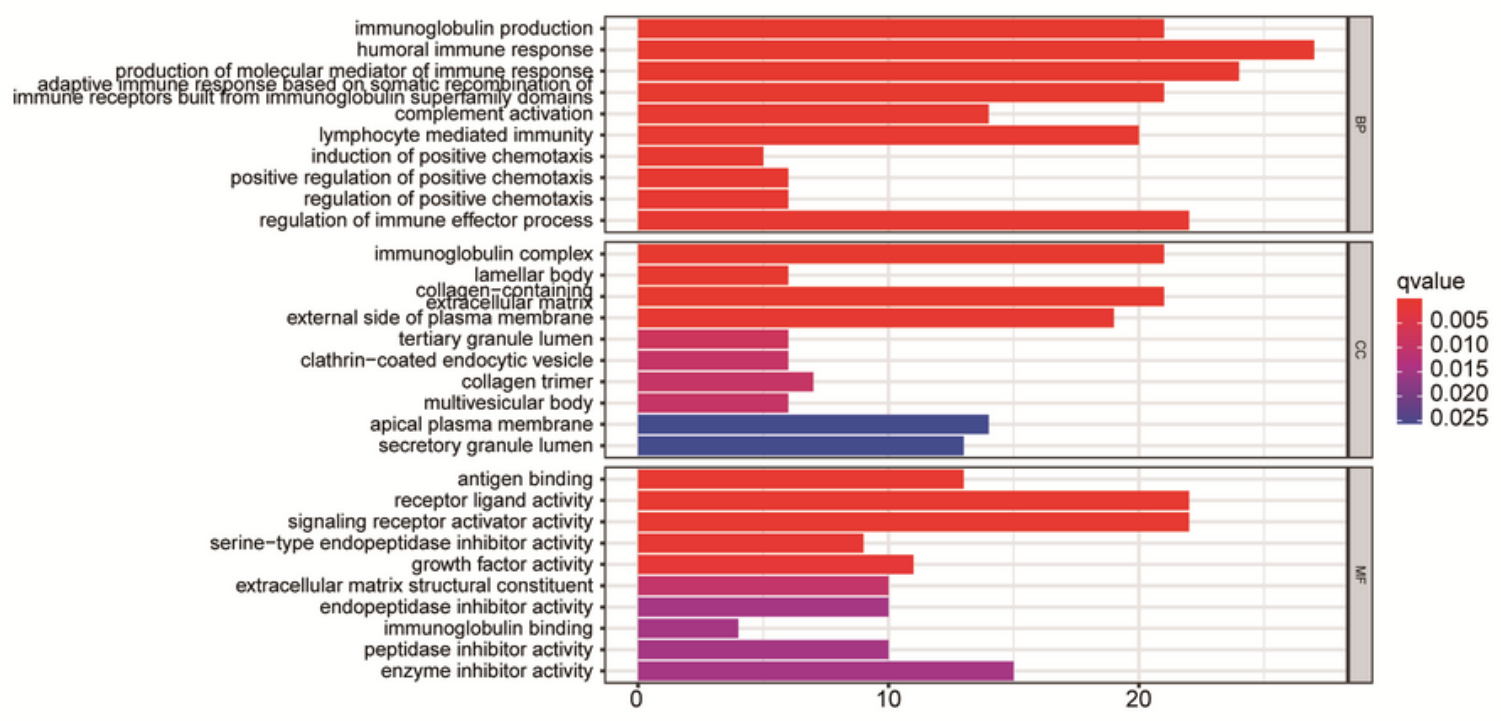

b

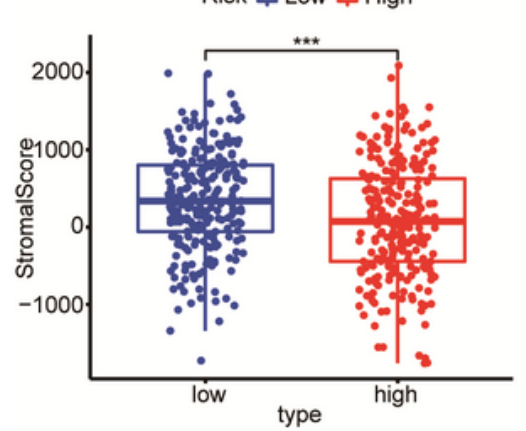

g
C

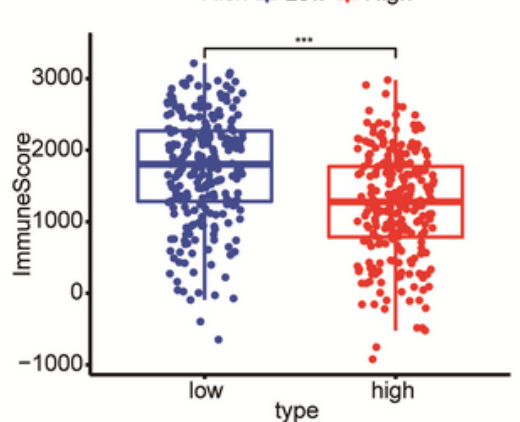

f

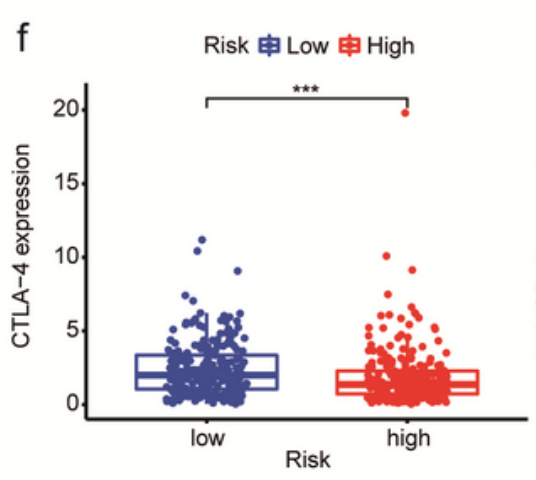

d

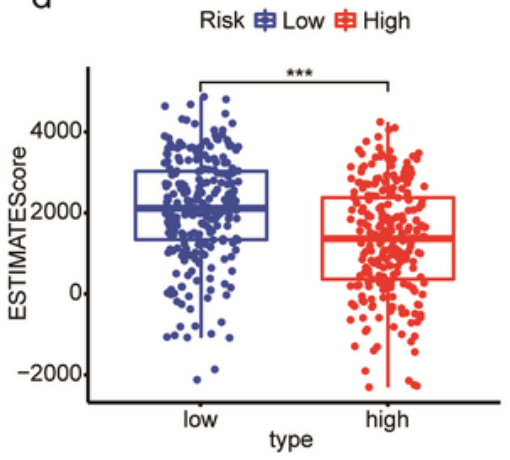

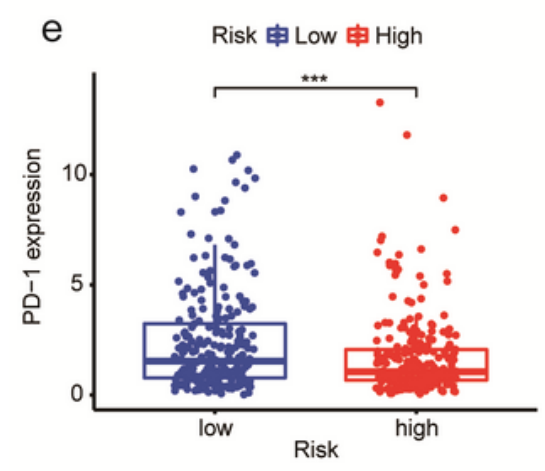

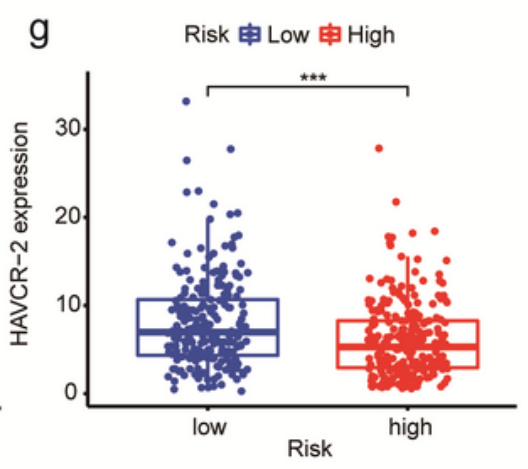

Figure 8 
Exploration of functional phenotypes and related tumor immunity of the oncosis-based prognostic signature. (a) GO enrichment analysis shows that the risk assessment model is closely associated with immune functions. (b-d) The box plots suggested that the StromalScore (b), ImmuneScore (c), and ESTIMATEScore (d) in low-risk patients were significantly higher than high-risk patients. (e-f) The box plots revealed that low-risk patients had a higher expression of PD-1 $(p>0.001)$, CTLA-4 $(p>0.001)$, and HAVCR-2 $(p>0.001)$.
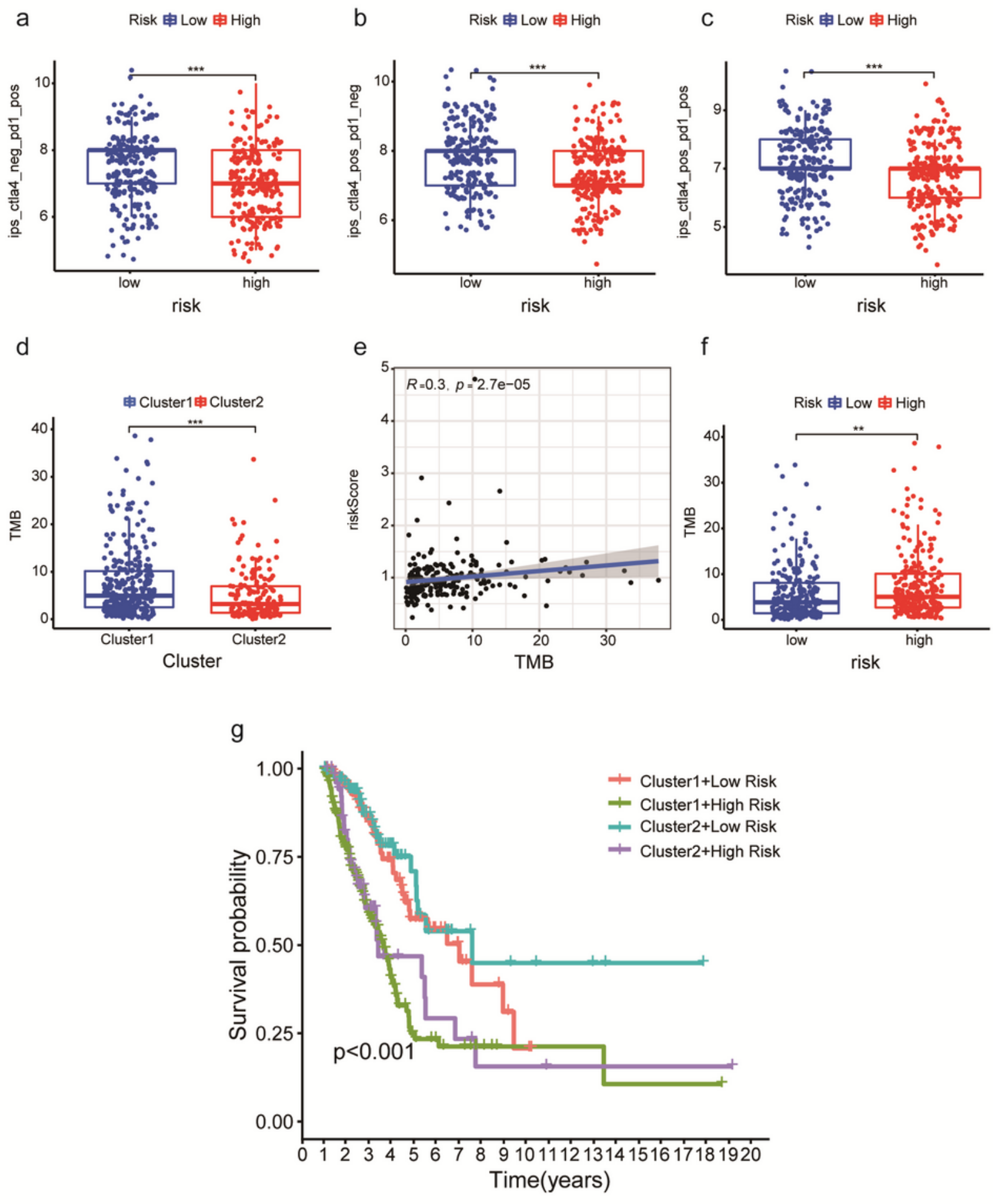


\section{Figure 9}

Exploration of the clinical significance of the oncosis-based prognostic signature. (a-c) The box plots suggest that low-risk patients have a relatively higher IPS of PD-1 positive (a), CTLA-4 positive (b), and CTLA-4 positive + PD-1 positive (c). (d) The box plots show that the TMB of patients in cluster1 is significantly higher than that of cluster2 patients. (e and f) The scatter plot (e) and the box plot (f) indicate that TMB is positively associated with the risk score, and LUAD patients with high-risk scores tend to have a higher TMB. (g) The survival curve reveals that low-risk patients in cluster2 have the best survival outcomes.

\section{Supplementary Files}

This is a list of supplementary files associated with this preprint. Click to download.

- SupplyMaterial.rar 